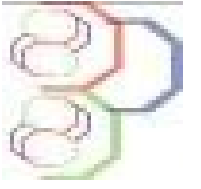

Journal of Applied Biosciences 154: 15905 - 15912

ISSN 1997-5902

\title{
Accumulation des sucres dans les tiges et les feuilles de différentes variétés de tournesol exposées à l'environnement salin
}

\author{
OUAZZANI Chadia ${ }^{*}$ et MOUSTAGHFIR Abdellah² \\ 1 : Laboratoire de Biochimie et de Biologie Moléculaire, Faculté de Médecine et de Pharmacie Rabat, Université \\ Mohammed -V de Rabat, Maroc ; \\ 2 : Laboratory of Research of Odontological Biomaterials and Nanotechnology. Faculté de Médecine Dentaire Rabat, \\ Université Mohammed - $V$ de Rabat, Maroc. \\ *Auteur correspondant : e-mail : ouazcom@yahoo.fr
}

Original submitted in on $31^{\text {st }}$ August 2020. Published online at www.m.elewa.org/journals/ on $31^{\text {st }}$ October 2020 https://doi.org/10.35759/JABs.154.7

\section{RESUME}

Introduction : Au Maroc, les périodes de sècheresse sont nombreuses et ont des conséquences néfastes sur la production agricole. Le tournesol est une plante oléagineuse très affectée par la sécheresse. Les plantes oléagineuses représentent $75 \%$ de la consommation d'huiles au Maroc.

Objectif: Dans les conditions de culture d'environnement salin, l'analyse des taux de sucres extraits à partir des tiges et des feuilles permet de révéler la corrélation de l'augmentation ou l'absence d'accumulation des sucres simples et la tolérance ou la sensibilité à la salinité.

Méthodologies et résultats : La culture de différentes variétés commerciales d'origine France et Maroc de tournesol révèle des pourcentages de diminution comprises entre $0 \%$ et $38 \%$ et entre $0 \%$ et $60 \%$ respectivement dans l'environnement de $\mathrm{NaCl} 60 \mathrm{mM}$ et de $\mathrm{NaCl} 120 \mathrm{mM}$ chez les différentes variétés étudiées. Les rapports de la teneur des sucres extraites des tiges et des feuilles dans les conditions de $\mathrm{NaCl}$ $60 \mathrm{mM}$ par rapport à la teneur sucres témoins et ceux de la teneur des sucres dans les conditions de $\mathrm{NaCl}$ $120 \mathrm{mM}$ par rapport à la teneur des sucres témoin sont compris entre 1,042 et 1,50 et entre 1,88 et 2,16 respectivement chez les différentes variétés étudiées.

Conclusion et Application des résultats : Ces variétés présentent une variabilité de la tolérance et la sensibilité. 2 variétés de tournesol (AG5640 et MK 3790) présentent une tolérance à l'environnement, 2 variétés de tournesol (Natil et Cliosol) présentent une tolérance modérée et 6 variétés (Santaffe, ORO 9, Albena, Santos, Florinne et Fleury) sont sensibles. Chez ces variétés étudiées l'accumulation de sucres simples dans les feuilles et les tiges dans l'environnement salin pourrait contribuer au maintien de l'équilibre osmotique au niveau cellulaire dans des conditions de déshydratation. D'autres études basées sur l'identification des paramètres génétiques et physiologiques permettront un criblage précoce de plantes adaptées à des contraintes liées aux stress salin et hydrique et classer les différentes variétés selon leur tolérance aux stress salin.

Mots clés : Sélection tournesol, stress salin, tiges, feuilles, sucre. 


\section{ABSTRACT}

Introduction: In Morocco, periods of drought are numerous and have negative consequences on agricultural production. Sunflower is an oilseed plant that very affected by drought. Oil plants represent $75 \%$ of oil consumption in Morocco.

Objective: Under conditions of cultivation in a saline environment, analysis of the levels of sugars extracted from stems and leaves reveals the correlation of the increase or absence of accumulation of simple sugars and the tolerance or sensitivity to salinity.

Methodologies and results: The cultivation of different commercial varieties of French and Moroccan origin of sunflower in a saline environment reveals percentages decrease in the fresh weight ranging between $0 \%$ and $38 \%$ and $0 \%$ and $60 \%$ respectively in the environment of $60 \mathrm{mM}$ and $120 \mathrm{mM}$ of $\mathrm{NaCl}$ in the different varieties studied. The ratios of the content of sugars extracted from the stems and leaves under the conditions of $60 \mathrm{mM} \mathrm{NaCl}$ conditions compared to the control sugar content and those of the content 120 $\mathrm{mM} \mathrm{NaCl}$ conditions compared to the control sugar content are between 1.042 and 1.50 and between 1.88 and 2.16 respectively in the different varieties studied.

Conclusion and Application of results: These varieties exhibit variability in tolerance and susceptibility. 2 varieties (AG5640 and MK 3790) show environmental tolerance, 2 varieties (Natil and Cliosol) show moderate and 6 varieties (Santaffe, ORO 9, Albena, Santos, Florinne et Fleury) are susceptible. In these varieties studied, the accumulation of simple sugars in leaves and stems in the saline environment could help maintain osmotic balance at the cellular level under dehydrated conditions. Further studies based on the identification of genetic and physiological parameters will allow an early screening of plants screening of plants adapted to constraints linked to salt and water stress to classify the different varieties according to their tolerance to salt stress.

Keywords: Sunflower selection, salt stress, stems, leaves, sugars

\section{INTRODUCTION}

Les plantes subissent l'action de divers stress liés à l'effet du climat selon lequel subsistent des températures extrêmes, basses et élevées. La sècheresse peut être causée par un environnement salin, le manque d'eau, des radiations et des concentrations salines; la salinité, la température et la lumière sont les principaux facteurs qui limitent le développement des plantes dans les régions arides ou semi arides, par leur influence sur l'absorption de l'eau et de certains ions toxiques ou non la plante $(F A O, 2015)$. Les effets du stress hydrique ont des répercussions sur la croissance et le développement des plantes liées à la réduction de la photosynthèse, de la division cellulaire en relation avec les déséquilibres hormonaux qui se sont installés (acide abscissique, cytoquinines). L'ensemble de ces phénomènes aboutissent à une diminution de qualité et quantité de productions végétales (Benaceur et al., 2001,Ould Mohamdi et al., 2011). Face à ces stress, les plantes développent plusieurs mécanismes de tolérance à la sécheresse salinité. L'échappement consiste à réaliser le cycle pendant la période favorable telle que la précocité, la dormance et la plasticité du développement. L'évitement de déshydratation permettant eu maintien du potentiel hydrique élevé dans la plante est réalisé par un maintien des quantités d'eau consommées ou une réduction des pertes d'eau par un ajustement osmotique, élasticité membranaire. La tolérance à la déshydratation consiste en un ensemble d'aptitude à résister aux effets d'un faible potentiel hydrique (résistance mécanique, dégradation membranaire. Dans des conditions de déshydratation L'équilibre osmotique cellulaire est maintenu par l'accumulation d'osmolytes (Bray et al., 2000). Chez le blé, traité par le sodium azide, des mutants de Tritium ont été identifiés pour leur grande aptitude à l'eau liée au niveau des feuilles, (Rascio et al., 1999). Chez les graminées et le tournesol les fructanes ont été étudiés en relation avec l'ajustement osmotique (Hincha et al., 2006). Les fructanes sont cités, comme étant impliqués dans la résistance aux stress environnementaux tels que 
le froid et la sécheresse (Vijn et al., 1999). Au Maroc, le tournesol est la principale oléagineuse annuelle et représente un pourcentage de $15 \%$ de la production totale d'huile. II couvre avec les autres espèces oléifères, moins de $30 \%$ des besoins. Le Maroc est tributaire des conditions climatiques lesquelles les périodes pluvieuses sont peu fréquentes. Les superficies cultivées de tournesol au Maroc sont 2,5\% de la superficie totale des plantes oléagineuses. Le principal facteur limitant l'eau disponible pour l'irrigation du tournesol entraine des conséquences sur le ralentissement du progrès économique. Beaucoup d'études ont été faites pour la création de variétés de tournesol face à la résistance aux agents pathogènes (phomopsis et mildiou) tout en gagnant sur la productivité et la teneur en huile (Vear et al., 2003). Par contre, les variétés de tournesol tolérantes à l'environnement salin n'ont pas été sélectionnées. Les essais effectués au préalable ont permis de donner des résultats variables liés à la contrainte de l'expérimentation dans les conditions moins astreignant (irrigation d'appoint, terrains profonds) (Jouffret et al., 2008). La sélection végétale a permis d'augmenter la production agricole et l'amélioration de la qualité technologique ou nutritionnelle des récoltes. La présente étude a eu pour objectif d'analyser les caractéristiques agronomiques de différentes variétés de tournesol commerciales d'origine France et des variétés locales d'origine Maroc cultivées dans les conditions normales et celles de différents environnements salins. Les marqueurs moléculaires liés à la tolérance à la salinité chez le tournesol à partir de matériel génétique constitué de populations d'hybrides ont été étudiés. La variabilité de la teneur des sucres solubles extraits des feuilles et des tiges des plantules suite à la réponse à l'adaptation des plantules durant la croissance dans différents environnements salins a été évaluée. L'amélioration de génotypes résistants aux contraintes de l'environnement représente un grand défi aux sélectionneurs de tournesol. La recherche de marqueurs génétiques et physiologiques corrélés à l'adaptation d'un stress hydrique et salin permet l'identification précoce des différentes variétés de tournesol selon leur tolérance à l'environnement salin.

\section{MATERIEL ET METHODES}

Matériel : Douze variétés de tournesol commerciales d'origine France et Maroc sont représentées sur le tableau 1.

Tableau 1 : Liste des différentes variétés de tournesol

\begin{tabular}{c|c|c}
\hline Variété & Origines & Caractéristiques \\
\hline AG 5640 & Agri Genetics, LG Seeds Semences Limagrain & Hybride \\
\hline Albena & RM PrograinGénétique, France / IWS, Bulgaria & Hybride simple précoce \\
\hline Cliosol & France & Hybride \\
\hline DK3790 & Gallix RAGT, France / GKI, Hongrie/ Dekalb, & Hybride \\
\hline Euroflor & Rustica Semences, France / Sigco, U.S.A & Hybride simple tardif \\
\hline Fleury & AS 477 ASGROW & Hybride \\
\hline Florine & AS 440 ASGROW & Hybride \\
\hline Karima Oro 9 & I.N.R.A MAROC & Population précoce \\
\hline Peredovik Vniimk & Russie & Population \\
\hline Santiago & Hilleshög-NK, France / ICCPT, Rumania & Hybride simple demi précoce \\
\hline Santaffe & France & Hybride \\
\hline SH 26 & Semillas Pacifico Espagne & Hybride trois voies \\
\hline
\end{tabular}


Culture des semences des différentes variétés de tournesol : Les graines ont été rincées avec l'eau distillée additionnée de $\mathrm{NaOCl}$ et mises en culture dans des bacs contenant du sable terreau (tourbe) et du solprovenant de la région de Rabat. Les plantules témoins sont arrosées avec l'eau d'assainissement urbain. Les plantules conditionnées à l'environnement salin sont irriguées avec la solution de $\mathrm{NaCl} 60 \mathrm{mM}$ et $120 \mathrm{mM}$.

\section{Méthodes}

Détermination des poids frais des plantules de tournesol : La moyenne des poids frais de 6 plantules de tournesol témoins et celles cultivées sous l'environnement salin est déterminée aux différents stades de développement des plantules pendant la durée de 40 jours.

Extraction des sucres à partir des feuilles et des tiges : $0,1 \mathrm{gr}$ de feuilles et de tiges sont prélevées séparément à partir des plantules témoins et celles cultivées dans l'environnement salin $60 \mathrm{mM}$ et $120 \mathrm{mM}$. Après rinçage à l'eau du robinet additionné de $\mathrm{NaOCl}$, les feuilles sont additionnées de $2 \mathrm{ml}$ de tampon Tris $\mathrm{pH}$ 8,6, cystéine $0,2 \%$, albumine $0,1 \%$, polyvinylpyrrolidone $1 \%$ et $\beta$-mercaptoéthanol $0,1 \mathrm{mM}$ et broyés dans un mortier à $4^{\circ} \mathrm{C}$. Après centrifugation.et élimination des débris cellulaires le surnagent est récupéré et est additionné de $2 \mathrm{ml}$ d'éthanol et chauffé à $80^{\circ} \mathrm{C}$ pendant $1 / 2 \mathrm{~h}$. Après centrifugation, le surnagent contenant les sucres réducteurs est récupéré et conservé pour le dosage des sucres solubles (Conroy et al., 1988).

Dosage des sucres solubles : Le dosage des sucres est effectuée par la méthode de Cooper et al., 1970. Une fraction de $100 \mu \mathrm{l}$ des extraits de sucres est additionnée de $5 \mathrm{ml}$ de la solution d'orthotoluidine. Après chauffage pendant 8 min à $100^{\circ} \mathrm{C}$, les solutions sont refroidies et l'absorbance de la coloration obtenue est déterminée à la longueur d'onde de $630 \mathrm{~nm}$. Les concentrations de glucose des extraits des tiges et des feuilles sont déterminées à partir d'une courbe d'étalonnage de glucose.

\section{RESULTATS}

Variation des poids frais des différentes variétés de plantules de tournesol cultivées dans un environnement salin

Tableau 2 : Variation des pourcentages de diminution des poids frais (PF) (gr) des plantules de tournesol cultivées dans un environnement de $\mathrm{NaCl} 60 \mathrm{mM}$ et $120 \mathrm{mM}$ par rapport à celles cultivées dans un environnement témoin (abs de $\mathrm{NaCl}$ ). T : témoin. $1: \mathrm{NaCl}: 60 \mathrm{mM}$. 2 : $\mathrm{NaCl} 120 \mathrm{MM}$.

\begin{tabular}{c|c|c|c|c|c|c|c}
\hline Variété & $\begin{array}{c}\text { DuréeAjout } \\
\mathbf{N a C l}\end{array}$ & $\begin{array}{c}\text { Total croissance } \\
\text { (jours) }\end{array}$ & $\mathbf{T}$ & $\begin{array}{c}\mathbf{N a C l ~ 6 0} \\
\mathbf{m M}\end{array}$ & $\begin{array}{c}\mathbf{N a C l ~ 1 2 0} \\
\mathbf{m M}\end{array}$ & $\begin{array}{c}\mathbf{P F} \% \mathbf{1}^{\mathbf{1}} \\
\mathbf{V}\end{array}$ & $\begin{array}{c}\mathbf{P F} \mathbf{\%}^{2} \\
\mathbf{V}\end{array}$ \\
\hline Santaffe & 25 & 40 & 1,18 & 0,7 & 0,5 & 28 & 48 \\
\hline ORO 9 & 25 & 40 & 1,3 & 0,62 & 0,4 & 37 & 60 \\
\hline AG 5640 & 30 & 50 & 1,2 & 1,2 & 1,2 & 0 & 0 \\
\hline Albena & 25 & 31 & 1,38 & 1,2 & 1,00 & 40 & 19 \\
\hline Florinne & 21 & 31 & 1,42 & 1,2 & 0,9 & 17 & 39 \\
\hline
\end{tabular}

Tableau 3 : Variation des pourcentages de diminution des poids frais (gr) des plantules de tournesol cultivées dans un environnement de $\mathrm{NaCl} 60 \mathrm{mM}$ et $120 \mathrm{mM}$ par rapport à celles cultivées dans un environnement témoin (absence de $\mathrm{NaCl}$ ).

\begin{tabular}{|c|c|c|c|c|c|c|c|}
\hline \multirow[t]{2}{*}{ Variété } & \multirow{2}{*}{$\begin{array}{l}\text { Durée } \\
\text { Stress }\end{array}$} & \multirow{2}{*}{$\begin{array}{c}\text { Total croissance } \\
\text { (jours) }\end{array}$} & \multicolumn{3}{|c|}{ Poids Frais } & \multirow{2}{*}{$\begin{array}{c}\text { PF \% } \\
\qquad \\
\end{array}$} & \multirow{2}{*}{$\begin{array}{c}\text { PF \% } \\
\qquad\end{array}$} \\
\hline & & & $T$ & $\begin{array}{c}\mathrm{NaCl} 60 \\
\mathrm{mM}\end{array}$ & $\begin{array}{c}\mathrm{NaCl} 120 \\
\mathrm{mM}\end{array}$ & & \\
\hline Santiago & 30 & 43 & 1,5 & 1,1 & 1,2 & 33 & 25 \\
\hline MK 3790 & 30 & 50 & 1,30 & 1,59 & 0,91 & 0 & 20 \\
\hline Cliosol & 30 & 43 & 0,94 & 0,91 & 0,58 & 3 & 38 \\
\hline Santos & 30 & 50 & 2,40 & 2,08 & 1,51 & 13 & 37 \\
\hline Natil & 26 & 43 & 1,44 & 1,21 & 1,13 & 16 & 21,5 \\
\hline Fleury & 26 & 43 & 1,94 & 1,53 & 1,40 & 21 & 28 \\
\hline
\end{tabular}


La variabilité des poids frais durant la croissance des plantules des différentes variétés de tournesol (Tableau $\mathrm{n}^{\circ} 2$ ) cultivées dans l'environnement de $\mathrm{NaCl} 60 \mathrm{mM}$ révèle un pourcentage de diminution des poids frais de $28 \%, 37 \%, 0 \%, 0 \%$ et $17 \%$ respectivement chez les variétés Santaffe, Oro 9, AG 5640, Albenna et Florinne. Les variétés AG 5640 et Albenna présentent une tolérance à l'environnement salin $\mathrm{NaCl} 60 \mathrm{mM}$ par rapport aux variétés Santaffe, Oro 9 et Florinne. Dans l'environnement de $\mathrm{NaCl} 120 \mathrm{mM}$, les pourcentages de diminution des poids frais sont $48 \%, 60 \%, 0 \%, 19 \%$ et $39 \%$ respectivement chez les variétés Santaffe, Oro 9, AG 5640, Albenna et Florinne. La variété AG 5640 présente une tolérance à l'environnement salin par rapport aux autres variétés étudiées. La variété Albena présente une tolérance à l'environnement salin $120 \mathrm{mM}$ par rapport aux variétés Sanatffe, ORO9 et Florine. Les variétés Santaffe, Oro9 et Florinne présentent une forte sensibilité à l'environnement $\mathrm{NaCl} 120 \mathrm{mM}$. La croissance des plantules de tournesol durant 50 jours (tableau 3) cultivées dans l'environnement de $\mathrm{NaCl} 60$ Mm révèlent des diminutions de poids frais de $34 \%, 0 \%$, $3 \%, 30 \%, 16 \%$ et $21 \%$ respectivement chez les variétés Santiago, MK 3790, Cliosol, Santos, Natil et Fleury. Les variétés Cliosol et MK 3790 sont tolérantes à l'environnement de $\mathrm{NaCl} 60 \mathrm{Mm}$ par rapport aux variétés Natil, Santos, Santiago et Fleury. A l'environnement de $\mathrm{NaCl} 120 \mathrm{Mm}$, les pourcentages de diminutions des poids frais sont $25 \%, 30 \%, 38 \%, 37 \%, 21 \%$ et $28 \%$ respectivement chez les variétés Santiago, MK 3790, Cliosol, Santos, Natil et Fleury. La variété MK 3790 présente une sensibilité modérée à l'environnement de $\mathrm{NaCl} 120 \mathrm{Mm}$ par rapport aux variétés Santiago, Cliosol, Santos, Natil et Fleury.

Variation de la teneur en sucres extraits à partir des tiges et des feuilles

Tableau 4 : Rapport $(R)$ des teneurs en sucres extraites à partir des tiges (1) de plantules de tournesol cultivées dans l'environnement de $\mathrm{NaCl} 60 \mathrm{mM}$ et $120 \mathrm{mM}$ par rapport aux teneurs des sucres des tiges témoins cultivées en absence de $\mathrm{NaCl}$.

\begin{tabular}{c|c|c|c|c|c}
\hline Variété & \multicolumn{3}{|c|}{ Teneur sucres des tiges gr/gr } & R NaCl & R NaCl \\
poids frais & $\mathbf{6 0 ~ m M}$ & $120 \mathrm{mM}$ \\
\hline & Témoin & $\begin{array}{c}\text { NaCl 60 } \\
\mathbf{m M}\end{array}$ & $\begin{array}{c}\mathrm{NaCl} \mathrm{120} \\
\mathbf{m M}\end{array}$ & & \\
\hline MK 3790 (1) & 0,27 & 0,35 & 0,52 & 1,50 & 2,16 \\
\hline Santiago (1) & 0,174 & 0,23 & 0,4 & 1,38 & 2,47 \\
\hline Cliosol (1) & 0,33 & 0,35 & 0,62 & 1,042 & 1,88 \\
\hline
\end{tabular}

Tableau 5 : Rapport $(R)$ des teneurs en sucres des Feuilles (2) des plantules de Tournesol cultivées dans l'environnement $\mathrm{NaCl} 60 \mathrm{mM}$ et $120 \mathrm{mM}$ par rapport aux teneurs des sucres des feuilles des plantules cultivées en absence de $\mathrm{NaCl}$.

\begin{tabular}{c|c|c|c|c|c}
\hline \multirow{2}{*}{ Variété } & \multicolumn{2}{|c|}{ Teneur sucres des feuilles (gr)/gr poids } & NaCl 60 mM & NaCl 120 mM \\
$\mathbf{R}$ & \multicolumn{2}{|c|}{ frais } & & \\
\hline & Témoin & $\begin{array}{c}\text { NaCl 60 } \\
\mathbf{m M}\end{array}$ & $\begin{array}{c}\text { NaCl 120 } \\
\mathbf{m M}\end{array}$ & & \\
\hline Santos (2) & 0,34 & - & 1,14 & - & 3,38 \\
\hline Natil(2) & 0,35 & - & 2,24 & - & 6,46 \\
\hline AG 5640 (2) & 0,52 & 0,66 & 0,53 & 1,26 & 1,021 \\
\hline SH 26 (2) & 0,4 & 0,51 & 0,46 & 2,46 & 1,15 \\
\hline Peredowich & 0,44 & 0,80 & 0,78 & 1,81 & 1,77 \\
\hline Albena & 0,44 & 0,606 & 0,98 & 1,37 & 2,22 \\
\hline
\end{tabular}


Les rapports de la teneur des sucres tiges $\mathrm{NaCl} 60 \mathrm{mM}$ par rapport à la teneur des sucres tiges témoins sont $1,50,1,38,1,042$ respectivement chez les variétés Santiago, MK 3790 et Cliosol (tableau 4). Les rapports de la teneur des sucres tiges $\mathrm{NaCl} 120 \mathrm{Mm}$ par rapport à la teneur des sucres tiges témoin sont 2,16, 2,47 et 1,88 respectivement chez les variétés Santiago, MK 3790 et Cliosol. Les différentes variétés répondent par l'augmentation de la teneur en sucres dans les conditions de l'environnement salin. Les rapports de la teneur des sucres extraits à partir des tiges chez les variétés Santiago, MK 3790 et Natil cultivées dans l'environnement de $\mathrm{NaCl} 120 \mathrm{mM}$ par rapport à la teneur des sucres tiges cultivées dans les conditions témoins sont plus élevés par rapport à ceux des conditions de l'environnement de $\mathrm{NaCl} 60 \mathrm{mM}$. L'augmentation de la teneur en sucres présente une variabilité chez les différentes variétés étudiées. La teneur en sucres extraites dans les tiges chez la variété Cliosol présente une légère augmentation dans l'environnement de $\mathrm{NaCl}$ $60 \mathrm{mM}$ par rapport à la teneur en sucres extraites dans l'environnement en absence de $\mathrm{NaCl}$. Cette variété présente une variabilité de la réponse de la teneur en sucres liée à l'adaptation à la salinité par rapport aux variétés Santiago et MK 3790. Les variétés MK 3790 et Santiago présentent une teneur en sucres extraites à partir des tiges plus élevés par rapport à la teneur en sucres des tiges témoins. Les deux variétés MK 3790 et Santiago présentent une augmentation modérée de la teneur en sucres liée à l'adaptation à l'environnement salin. Les rapports des teneurs des sucres extraits des

\section{DISCUSSION}

La salinité, la présence de concentration élevée en chlorure de sodium limite la culture des variétés de Tournesol. Les différentes variétés étudiées présentent une variabilité liée à la sensibilité ou la tolérance à la salinité. Les variétés tolérantes à la salinité sont d'origine France AG 5640 et hybride simple (Albena) présentent une tolérance à la salinité supérieure à celles des variétés populations d'origine Maroc, la population Russe (Vear et al., 2011). La variété hybride simple demie précoce Santiago et la variété Santaffe présentent une forte sensibilité aux concentrations de $\mathrm{NaCl} 60$ et $120 \mathrm{mM}$. Les variétés Natil et Fleury présentent une sensibilité modérée à l'environnement salin de $\mathrm{NaCl} 60 \mathrm{mM}$ et $120 \mathrm{mM}$. Les répercussions des stress sur les plantes dépendent du stade développement du mode de culture et du climat (Maury et al., 2011). Les réductions des poids frais au cours de la croissance des plantules de Tournesol dans l'environnement salin $120 \mathrm{Mm}$ sont plus élevées par feuilles $\mathrm{NaCl} 60 \mathrm{mM}$ par rapport à la teneur des sucres feuilles témoins sont $1,26,2,46,1,81$ et 1,37 respectivement chez les variétés AG 5640, SH 26 , Peredowich et Albena (tableau 5). Les rapports des teneurs de sucres feuilles $\mathrm{NaCl} 120 \mathrm{mM}$ par rapport à la teneur des sucres feuilles témoin sont 1,021, 1,15, 1,77 et 2,22 respectivement chez les variétés AG $5640, \mathrm{SH}$ 26, Peredowich et Albena. Les rapports des teneurs des sucres des feuilles $\mathrm{NaCl} 120 \mathrm{Mm}$ par rapport à la teneur des sucres feuilles témoin sont 3,38 et 6,46 respectivement chez les variétés Santos et Natil. Le rapport de la teneur en sucres feuilles $\mathrm{NaCl} 60 \mathrm{mM}$ par rapport à la teneur des sucres feuilles témoin de la variété SH 26 est plus élevé par rapport à celui des variétés AG5640, Peredowich et Albena. Le rapport de la teneur en sucres des feuilles $\mathrm{NaCl} 120 \mathrm{Mm}$ par rapport à la teneur des sucres feuille témoin des variétés Natil et Santos est plus élevé par rapport à celui des variétés AG 5640, SH 26, Peredowich et Albena. La variété AG 5640 ne présente pas d'augmentation de sucres extraits dans les feuilles prélevées dans les conditions d'environnement de $\mathrm{NaCl} 60 \mathrm{mM}$ et $120 \mathrm{mM}$ par rapport à la teneur en sucres extraits à partir des feuilles dans les conditions témoins. La variété MK 3790 présente une meilleure tolérance à la croissance à dans les conditions de l'environnement de $\mathrm{NaCl} 60 \mathrm{mM}$ et a un rapport de sucres dans les tiges plus élevé par rapport à celui des variétés Santiago et Natil. La variété AG5640 a une faible augmentation de sucres dans l'environnement 60 $\mathrm{mM}$ et $120 \mathrm{Mm}$ et présente une tolérance à la croissance dans l'environnement salin.

rapport celles de l'environnement $60 \mathrm{mM}$. Les travaux d'Aziadekey et al., (2014) ont révèle la réduction des caractères agromorphologiques chez une lignée de Niébé cultivé dans un environnement de stress hydrique pendant une période de 16 jours. Dans les conditions de culture d'environnement salin, l'analyse des taux de sucres extraits à partir des tiges et des feuilles révèle une corrélation entre la réponse à la plante par l'augmentation ou l'absence d'accumulation des sucres simples lié à la tolérance à la salinité et la sensibilité à la salinité chez les variétés AG 5640, Albena et Cliosol au cours de la croissance des plantules de tournesol. Le rapport de la teneur en sucres tiges $\mathrm{NaCl} 120 \mathrm{mM}$ par rapport à la teneur des sucres tiges témoins est plus élevé par rapport à celui des sucres tiges $\mathrm{NaCl} 60 \mathrm{mM}$ des sucres témoin. Chez les feuilles cette augmentation n'est pas observée chez les différentes variétés étudiées. Chez la variété SH 26 la teneur est diminuée, chez la variété Pederowich elle est peu modifiée. 
L'accumulation faible des sucres simples dans les feuilles et la corrélation à la tolérance à l'environnement salin chez la variété AG 5640 pourrait être liée à un mécanisme d'ajustement osmotique différent par rapport aux autres variétés étudiées. Aux stress de $\mathrm{NaCl} 120$ $\mathrm{mM}$, le taux de sucres extraits des tiges est plus élevé par rapport aux taux de sucres extraits des tiges conditionnées aux stress $60 \mathrm{mM}$. Les mécanismes d'adaptation aux contraintes hydriques présentent une variabilité génétique chez les espèces cultivées. Ces résultats révèlent la grande diversité des génotypes de tournesol face à leur comportement dans un environnement salin (Maury et al., 2011). La réduction de la production végétale sous l'influence d'un environnement salin caractérisée par les modifications morphologiques, physiologiques et biochimiques a été mis en évidence par différents auteurs (Tafforeau, 2002, Ashraf et al., 2004). L'étude de Gogbeu et al., 2019 a révélé la teneur en sucres au niveau des feuilles ne dépassant pas l'âge de 90 jours plus élevée par rapport à celle des racines de la variété zouhn-kinmin (Gogbeu

\section{CONCLUSION}

Ces résultats révèlent la grande diversité des génotypes de tournesol face à leur comportement dans un environnement salin. Deux variétés commerciales présentent une tolérance à l'environnement salin total, deux variétés présentent une tolérance modérée et six variétés sont très sensibles par rapport à douze variétés étudiées. La synthèse d'osmolytes permet le maintien de l'équilibre osmotique cellulaire dans les conditions de stress salin. La grande variabilité caractérisée chez les

\section{REFERENCES BIBLIOGRAPHIQUES}

Ashraf M., Harris P. J. C., 2004. Potential biochemical indicators of salinity tolerance in plants. Plant Science, 166: 3-6. DOI: 10.1016/i.plantsci.2003.10.024

Aziadekey M., Atayi A., Odah A., Magamana A. E., 2014. Etude de l'influence du stress hydrique sur deux lignées de niébé. European Scientific Journal. vol.10, No.30 : 328-338.

Benaceur M., Rahmoun C., Sdiri H.,Medahi M.,Selmi M., 2001. Effet du stress salin sur la germination, la croissance et la production de grains de blé. Sécheresse, 12 (3): 167- 174.

Blumwald E., Grover A., Good A.G., 2004. Breeding for abiotic stress resistance: challenges and opportunities. "New directions for a diverse planet». Proceedings of the $4^{\text {th }}$ International Crop Science Congress, 26 September - 1 et al., 2019). Une accumulation de fructanes est également observée en réponse à la déshydratation chez de nombreuses espèces. Ces derniers jouent un rôle déterminant avec le saccharose dans l'ajustement osmotique, et aussi au niveau de la protection des membranes (Hinchaet al., 2007). Les molécules osmoprotectantes jouent le rôle de stabilisation membranaire (Folkert et al., 2001). La réponse à l'adaptation chez les plantes à la salinité s'accompagne de nouvelles voies métaboliques. Ces mécanismes installent la compartimentation vacuolaire ou l'exclusion des ions toxiques (Blumwald et al., 2004, Munns et al., 2008). L'excès du sodium dans le cytoplasme est expulsé vers le compartiment extra cellulaire ou au cloisonnement des ions $\mathrm{Na}^{+}$et $\mathrm{Cl}$ au niveau cellulaire et intracellulaire pour éviter l'augmentation de leurs concentrations dans le cytoplasme et des cellules de mésophile. La teneur en sucres augmente avec l'augmentation de la concentration du sel dans le milieu (Gogbeu et al. 2019).

différentes espèces de tournesol étudiées constitue des outils de sélection de génotypes adaptés aux stress salins. D'autres études basées sur l'investigation des facteurs agronomiques, génétiques et physiologiques permettront de distinguer la variabilité des plantes adaptées aux contraintes liées aux stress salin et hydrique et de classer les différentes variétés selon leur tolérance aux stress salins.

October, Brisbane, Australia.[CDROM].Web site www.cropscience.org.au.

Bray E. A., Bailey-Serre J., Weretilnyk E., 2000. Responses to abiotic stresses. Biochemistry and Molecular Biology of Plants. (eds B B Buchnau, W. Gruissem R. L. Jones, pp.11581203. American Society of Plant Physiologists, Rockville, MD.

Conroy J.P., Virgona J.M., Smillie R.M., Barlow E.W., 1988. Influence of drought acclimation and $\mathrm{CO}_{2}$ enrichment on osmotic adjustment and chlorophyll a fluorescence of sunflower during drought. Plant Physiology 86, 1108-1115.

Cooper G. R. and McDaniel V. 1970. The determination of glucose by the ortho-toluidine method (filtrate and direct procedure). Standard Methods of clinical chemistry. 61:159-170. 
FAO, 2015. État des ressources en sols du monde résumé technique, http://www.fao.org/3/ai5126f.pdf

Folkert A. H., Golovina E. A., Buintik J., 2001. Mechanisms of plant dessication tolerance. Trends in plant science 6 (9): 431-438.DOI: https://doi.org/10.1016/S1360-1385(01)02052$\underline{0}$.

Gogbeu S. J. , Yapo S. E. S., Gore B. B. N., Ayolie K, Kouass IN'Dri J., Nacanabo R., Dogbo D. O., Kouadio Y. J., 2019. Effets du traitement des plants de riz au chlorure de sodium sur la synthèse et accumulation des sucres totaux et des composés phénoliques ethano-solubles dans les feuilles et racines. Journal of Applied Bioscience, 135 : 13775 - 13787 https://www.ajol.info/index.php/jab/article/view/ 186360.

Hincha D.K., Popova A.V., Cacela C., 2006."Effects of sugars on the stability and structure of lipid membranes during drying", A. Leitmannova Liu ed., advances in planar lipid bilayers and liposomes, Elsevier, Amsterdam, 3: 189-217. DOI: 10.1016/S1554-4516(05)03006-1.

Hincha D.K., Livingston D.P., Premakumar R., Zuther E., Obel N., Cacela C., Heyer A.G. 2007."Fructans from oat and rye: composition and effects on membrane stability during drying", Biochimica and Biophysica Acta, 1768, 1611-1619. https://doi.org/10.1016/j.bbamem.2007.03.011

Jouffret P.,Vogrincic C., 2008. Description des freins et enjeux de production de tournesol, colza et soja à l'horizon 2009-2012 dans le Sud-Ouest. Rapport interne CETIOM, $27 \mathrm{p}$

Maury P., Langlade N., Grieu P., Rengel D., Sarrafi A., Debaeke P., Vincourt P. 2011. Ecophysiologie et génétique de la tolérance à la sécheresse chez le tournesol. Innovations Agronomiques. 14, 123-138.

Munns R.,Tester M., 2008. Mechanisms of salinity tolerance. Annual Review of Plant Biology, 59(1): 651-681. DOl: 10.1146/annurev.arplant.59.032607.092911.

Ould Mohamdi M., Bouya D., Ould Mohamed Salem A., 2011. Etude de l'effet du stress salin $(\mathrm{NaCl})$ chez deux variétés de tomate (Campbell 33 et Mongal). International Journal of Biological and Chemical Sciences. 5(3): 860-900. DOI: 10.4314/ijbcs.v5i3.72171

Rascio A., Russo M, Platani C., Giampaolo Ronga G., Di Fonzo N., 1999. Mutants of durum wheat with alterations in tissue affinity for strongly bond water. Plant Science. 144 (1): 25-34. https://doi.org/10.1016/S0168-9452(99)00053$\underline{9}$

Shawquat Md., Hamid A., Salahuddin A.B. M., Quasem A. 2008. Effect of sodium chloride on growth, photosynthesis and mineral ions accumulation of different types of rice (Oryza sativa). Journal Agronomy and Crop Science: 179(3): 149-161. DOI: 10.1111/j.1439-037X.1997.tb00511.X.

Tafforeau M., 2002. Etude des phases précoces de la transduction des signaux environnementaux chez le lin : une approche protéomique. Thèse de doctorat en Biochimie végétale. Université de Rouen. France. 255p.

Vear F., Bony Y., Joubert G., Tourvieille de Labrouche D., 2003. 30 years of sunflower breeding in France. OCL, 10 (1):66-73. DOI: 10.1051/ocl.2003.0066

Vear F., Muller M.H., 2011. Progrès variétal chez le tournesol : l'apport des ressources génétiques au sein du genre Helianthus Innovations Agronomiques, 14 : 139-150.

Vijn I, Smeekens S.1999. Fructan: more than a reserve carbohydrate. Plant Physiology. 120(2): 351360. DOI: $\underline{10.1104 / p p .120 .2 .351}$ 